\title{
Second-Line Capecitabine and Oxaliplatin Combination for Gemcitabine-Resistant Advanced Pancreatic Cancer
}

\author{
Ibrahim Vedat Bayoglu', Umut Varol' ${ }^{1}$, Ibrahim Yildiz ${ }^{1}$, Ugur Muslu², Ahmet \\ Alacacioglu $^{1 *}$, Yuksel Kucukzeybek ${ }^{1}$, Murat Akyol' ${ }^{1}$, Lutfiye Demir ${ }^{1}$, Ahmet \\ Dirican', Suna Cokmert', Yasar Yildiz ${ }^{1}$, Bulent Karabulut ${ }^{2}$, Ruchan Uslu², \\ Mustafa Oktay Tarhan ${ }^{1}$
}

\begin{abstract}
Background: The role of second-line therapy in metastatic pancreatic cancer is not clear. In this study, we aimed to explore the second-line efficiency of capecitabine and oxaliplatin (XELOX) in patients with advanced pancreatic cancer who have received gemcitabine-based first-line therapy. Materials and Methods: We retrospectively evaluated 47 patients with locally advanced or metastatic pancreatic cancer previously treated with gemcitabine-based first-line regimens. Treatment consisted of oxaliplatin $130 \mathrm{mg} / \mathrm{m}^{2}$ and capecitabine 1000 $\mathrm{mg} / \mathrm{m}^{2}$ twice daily with a 3 week interval, until unacceptable toxicity or disease progression. Results: Median number of cycles was 4 (range, 2-10). The overall disease control rate was 38.3\%. The median overall survival and progression-free survival from the start of second-line therapy were 23 weeks (95\% CI: 16.6-29.5 weeks) and 12 weeks (95\% CI: 9.8-14.4 weeks), respectively. The most common grade 3-4 toxicities were nausea, vomiting and hematologic side effects. Conclusions: Our result suggests that the combination of capecitabine and oxaliplatin was tolerated with manageable toxicity and showed encouraging activity as second-line treatment of advanced or metastatic pancreatic cancer patients with ECOG performance status 0-2.
\end{abstract}

Keywords: Capecitabine - oxaliplatin - advanced pancreatic cancer - second-line treatment

Asian Pac J Cancer Prev, 15 (17), 7119-7123

\section{Introduction}

Pancreatic adenocarcinoma is the seventh most common cancer and represents about $3 \%$ of all new cancer diagnoses. Furthermore, the mortality from pancreatic cancer accounted for 4-6\% of all cancer related deaths, which were ranked in the sixth place. Pancreatic cancer incidence and mortality rates have shown a gradual upward trend (Chen et al., 2013; Siegel et al., 2013; Zahir et al., 2013). Patients are mostly diagnosed with locally advanced or metastatic disease at first presentation. Despite recent advances in therapeutics, the prognosis of patients with advanced pancreatic cancer has remained poor (Canyilmaz et al., 2013). Although stage and surgical resection are the most important predictor factors for survival, only $10-15 \%$ of patients have localized or resectable disease at diagnosis (Qureshi et al., 2011). Also studies have determined many factors that affects on survival outcome of the patients (Luke et al., 2009; Cheung et al., 2013)

Systemic therapy is the mainstay of treatment for metastatic disease (Tokh et al., 2012). Several randomized trials have demonstrated that systemic chemotherapy prolongs survival and improves quality of life compared with best supportive care alone (Glimelius et al., 1996; Von Hoff et al., 1998). Gemcitabine is the primary cytotoxic drug in first-line treatment of pancreatic cancer (Burris et al., 1997; Inal et al., 2012). However, chemotherapy options are limited for patients relapsing following firstline gemcitabine-based treatment (Demols et al., 2006; Reni et al., 2006; Ignatiadis et al., 2006; Boeck et al., 2007; Kulke et al., 2007). So, the role of second-line therapy remains controversial (Choi et al., 2014).

Oxaliplatin is active in several solid tumor types especially in gastrointestinal cancers such as colorectal, gastro-esophageal and biliary cancers. When it is combined with gemcitabine, capecitabine or 5-Fluorouracil (5-FU), synergistic effects have been observed (Faivre et al., 1999; Díaz-Rubio et al., 2002). 5-FU has been used in treatment of metastatic pancreatic cancer patients for several decades and capecitabine is an oral pro-drug of 5-FU. Oxaliplatin and capecitabine combination is an effective and welltolerated regimen for metastatic colorectal cancer, but there is limited experience for treatment of pancreatic

${ }^{1}$ Department of Medical Oncology, Izmir Katip Celebi University Ataturk Training and Research Hospital, ${ }^{2}$ Department of Medical Oncology, Tulay Aktas Oncology Hospital, School of Medicine, Ege University, Izmir, Turkey*For correspondence:dralaca2000@ yahoo.com 
cancer. Besides, both oxaliplatine and capecitabine have been used as components of front-line therapy for metastatic pancreatic cancer (Conroy et al., 2011; Choi et al., 2012). Taken together, the antitumor activity of capecitabine and oxaliplatin combination in second-line treatment is not clear.

The purpose of this study was to evaluate the efficiency of palliative chemotherapy including capecitabine and oxaliplatine (XELOX) for patients with advanced pancreatic cancer that has progressed after gemcitabinebased treatments.

\section{Materials and Methods}

\section{Patient eligibility criteria}

Patients with histologically confirmed adenocarcinoma of the pancreas who have locally advanced or metastatic disease and who have progressed after gemcitabinebased regimens were included. Other inclusion criteria were Eastern Cooperative Oncology Group (ECOG) PS of 0 to 2 (Oken et al., 1982) and adequate hepatic, renal, and marrow function. Patients were excluded, if they had uncontrolled concurrent illnesses, previously received oxaliplatin or capecitabine, had gastrointestinal dysfunction or were unable to take oral medications.

Oxaliplatin was given intravenously on day 1 and capecitabine was administered orally twice daily for 14 days. A cycle of therapy was defined as 21 days. Tumor restaging was performed every 9 weeks (after 3 cycles). Oxaliplatin dose was $130 \mathrm{mg} / \mathrm{m} 2$ and the capecitabine dose was $1000 \mathrm{mg} / \mathrm{m} 2$ twice daily (total daily dose of 2000 $\mathrm{mg} / \mathrm{m} 2$ ). Treatment was administered until the disease progression, unacceptable toxicity or rejection of their treatment continuation. In patients aged $>65$ years, or with ECOG PS of 2 who having significant co-morbidities, the treatment was started with\%25 dose reduction and following first cycle chemotherapy treatment, patients without any grade 3-4 toxicity or intolerance to the treatment, the dose of the drug was increased to its standard dosage.

\section{Treatment endpoints}

Progression free survival (PFS) was the investigated primary endpoint, which was defined as the time from the start of second-line XELOX treatment to the first documentation of progression. First documentation of progressive disease (PD) was based on the definition of PD in the RECIST (Response Evaluation Criteria in Solid Tumors 1.1) guidelines (Eisenhauer et al., 2009), death as a result of any cause in the absence of previously documented PD and the investigator's clinical judgment of PD. We censored the last clinical visit data for patients that died without known progression. Overall survival (OS) was measured from the initiation of second-line XELOX treatment to death or to the last follow-up assessment. Hematologic and non-hematologic toxic effects were graded according to the Common Terminology Criteria for Adverse Events v3.0 (CTCAE v3.0) (Trotti et al., 2003).

\section{Response evaluation}

Evaluation of response assessment after and during treatment consisted of laboratory studies, including hematologic and biochemical profiles; computed tomography (CT) or magnetic resonance imaging (MRI) of the abdomen and pelvis. Response evaluation (CT or MRI) was performed during and after the treatment at every 9 weeks (3 cycles of chemotherapy). In patients with suspected clinical progression, response evaluation was performed earlier. Patients who received at least two doses of treatment were evaluable for response and patients who received one dose of the drugs were evaluable for toxicity. Toxicity analysis was carried out regarding the highest grade recorded. The results of carbohydrate antigen (CA 19-9) levels were recorded before the first therapy of XELOX.

\section{Statistical analysis}

Quantitative data are presented as the means, standard errors, medians, minimums and maximums; the results of qualitative analyses are presented as frequencies and percentages. The survival analysis and curves were established according to the Kaplan-Meier method and compared with the log-rank test. SPSS for Windows version 18.0 (SPSS Inc., Chicago, IL., USA) was employed for the data analysis.

\section{Results}

\section{Patient characteristics}

From June 2009 to December 2013, totally of 228 metastatic pancreas cancer patients were retrospectively evaluated. There were 47 patients treated with secondline XELOX after first-line gemcitabine-based treatment failure. The sample comprised 34 males (72.3\%) and 13 females $(27.7 \%)$. Median age of the patients was 60 years (range, 37-76 years). Thirty-one patients had an ECOG PS of 0 to $1(66 \%)$, while the rest had PS of $2(34 \%)$.

\section{Treatment details}

Thirty-four patients $(72.3 \%)$ were diagnosed at metastatic stage. Seventeen patients were initially operated; although $13(76.5 \%)$ of them had undergone pancreaticoduodenectomy or distal pancreatectomy, only surgical margins of 9 patients were negative for malignancy and $4(23.5 \%)$ of the operations were palliative surgical procedures. The median disease free survival was 3 months for patients who had been operated. Among the patients, $8(17 \%)$ were previously received adjuvant chemoradiotherapy and/or adjuvant chemotherapy. The median number of cycles for first-line gemcitabine-based treatment was 6 (range, 2-19) and for second-line XELOX therapy was 4 (range, 2-10). Treatment was ongoing in 3 patients $(6.5 \%)$ at the time of analysis. Demographic data of the patients and treatment details are shown in Table 1.

\section{Clinical outcome}

The patients were evaluated for their response to treatment (Table 2). Nearly half of the patients $(n=18$, $38.3 \%$ ) had disease control with chemotherapy. Only 8 patients $(17 \%)$ had partial response (PR) and 10 patients $(21.3 \%)$ had stable disease with XELOX treatment. No complete response was observed. Median progression free 
Table 1. Patient Characteristics

\begin{tabular}{|c|c|c|c|}
\hline \multicolumn{2}{|c|}{ Characteristics } & \multicolumn{2}{|r|}{$(\%)$} \\
\hline \multicolumn{4}{|c|}{ Clinical findings } \\
\hline \multirow[t]{2}{*}{ Sex } & Female & 13 & $(27.7)$ \\
\hline & Male & 34 & $(72.3)$ \\
\hline \multirow[t]{2}{*}{ Age (years) } & Median & 60 & \\
\hline & Range & 37-78 & \\
\hline \multicolumn{4}{|c|}{ ECOGa Performance status } \\
\hline & 0 & 8 & (17) \\
\hline & 1 & 23 & (49) \\
\hline & 2 & 16 & (34) \\
\hline \multirow{2}{*}{ Ca 19-9 } & Median & 1293 & \\
\hline & Range & $3-77.0$ & \\
\hline \multirow[t]{2}{*}{ Treatment } & Prior adjuvant chemotherapy & 8 & $(17)$ \\
\hline & Prior radiotherapy & 7 & (15) \\
\hline \multirow[t]{3}{*}{ Prior surgery } & No surgery & 30 & $(64)$ \\
\hline & R0 & 9 & (19) \\
\hline & $\mathrm{R} 1-2$ & 8 & (17) \\
\hline \multicolumn{4}{|c|}{ Response to first-line chemotherapy } \\
\hline & Median number of cycles (range) & ) 6 & $(2-19)$ \\
\hline & CR-PR & 9 & (19) \\
\hline & SD & 12 & $(26)$ \\
\hline & PD & 26 & (55) \\
\hline \multicolumn{4}{|c|}{ Number of XELOX cycles } \\
\hline & Median & 4 & \\
\hline & Range & $2-10$ & \\
\hline
\end{tabular}

Table 2. Treatment Characteristics and Efficacy of XELOX

\begin{tabular}{lcc}
\hline & No. of patients & $\%$ \\
\hline Response to XELOX & & \\
Objective response & 8 & 17.0 \\
Complete response & 0 & 0.0 \\
Partial response & 8 & 17.0 \\
Stable disease for $\geq 3$ months & 10 & 21.3 \\
Disease control rate & 18 & 38.3 \\
Median PFSa, weeks (95\% CI) & 12 & $(9.8-14.4)$ \\
Median OSb, weeks (95\% CI) & & \\
For all patients & 23 & $(16.6-29.5)$ \\
ECOG PS 0-1 & 26 & $(15.7-36.1)$ \\
ECOG PS 2 & 13.8 & $(6.5-21.3)$ \\
\hline
\end{tabular}

aprogression free survival, ${ }^{\mathrm{b}}$ overall survival

survival was 12 weeks (95\% CI 9.8-14.4 weeks; Figure 1) and median overall survival was 23 weeks $(95 \% \mathrm{CI}$ : 16.6-29.5 weeks; Figure 2) with a 6-month survival rate of $40 \%$. In univariate analysis, patients with ECOG PS 2 had shorter OS (13.8 weeks; 95\%CI: 6.5-21.3 weeks) than patients with ECOG PS 0-1 (26.0 weeks; 95\%CI: 15.7-36.1 weeks) ( $p=0.05$; Figure 3). Except ECOG PS, in univariate analysis, there was no correlation between other demographic parameters [initial staging (local vs metastatic), primary tumor resection (present $v s$ not), organ involved like lung or liver (present $v s$ not), sex (male $v s$ female), age $(>60 v s<60)]$ and median OS of the patients treated with second-line XELOX.

When we analyzed OS time for all patients, from the start of first-line treatment, we found as 53.7 weeks. (95\%CI: 44.0-63.4). According to their response to first-line treatment, the median survival was 57 weeks (95\%CI: 23-116) for gemcitabine responders (patients with a complete or partial response) and 30 weeks (95\% CI:

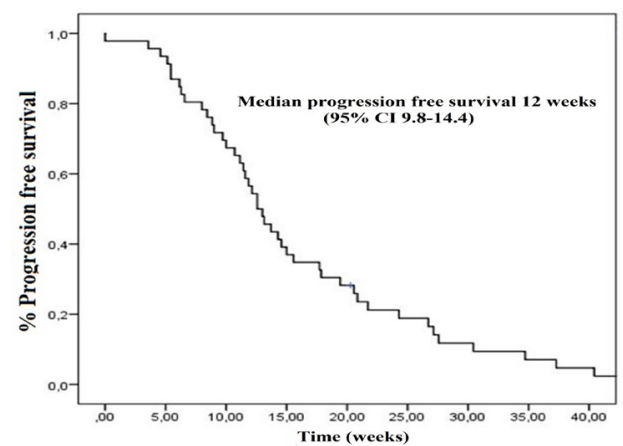

Figure 1.Progression free Survival Curve of the Patients Treated with Second-Line XELOX Chemotherapy

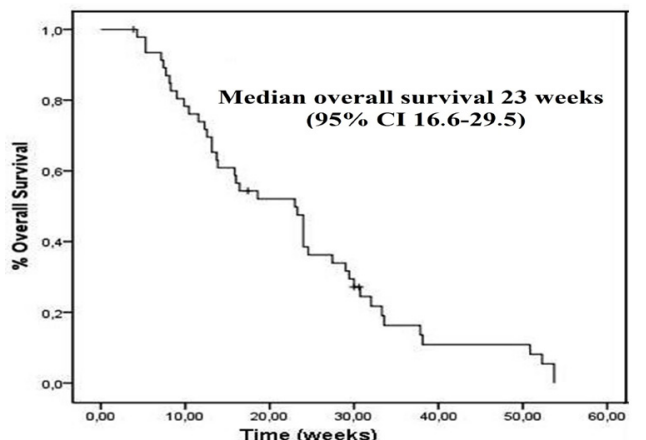

Figure 2. Overall Survival Curve of the Patients Treated with Second-Line XELOX Chemotherapy

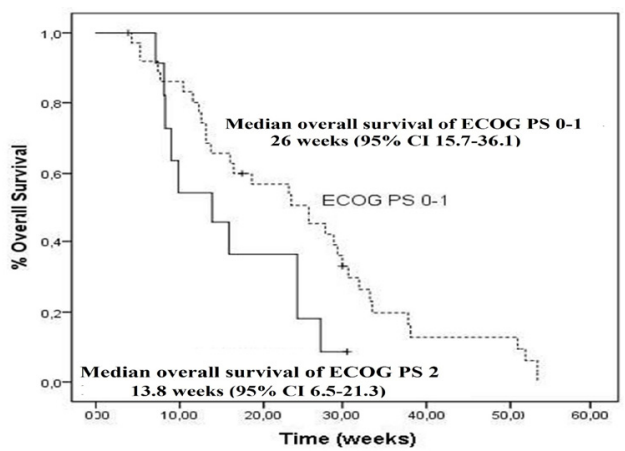

Figure 3. Overall Survival Curves of the Patients with ECOG PS 0-1 $\leq$ and $>2(p=0.05)$

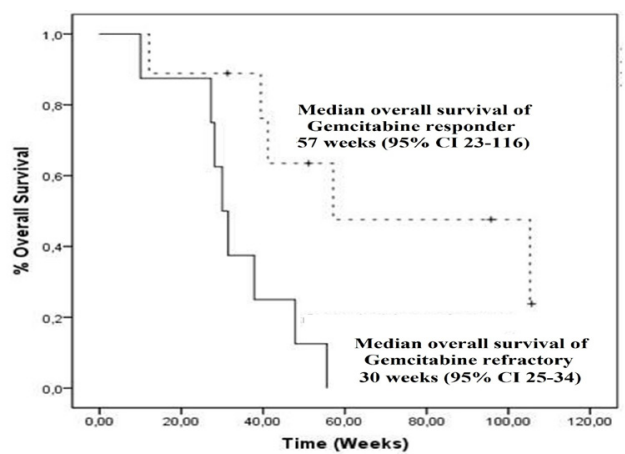

Figure 4. Overall Survival Curves of the Patients According to Response to the First-Line GemcitabineBased Chemotherapy

25-34, Figure 4) for gemcitabine refractory patients (patients with stable or progressive disease). There was a statistically significant difference between these groups $(\mathrm{p}=0.007)$. Baseline CA 19-9 data were available for 35 patients and the median CA 19-9 level was $1293 \mathrm{U} / \mathrm{mL}$ 
Table 3. Hematologic and Non-Hematologic Toxicity Profiles

\begin{tabular}{lrccccc}
\hline Toxicity & \multicolumn{5}{c}{ NCIa worst toxicity } \\
& Grade $1-2$ & Grade 3-4 & \multicolumn{2}{c}{ All } \\
& $\mathrm{n}$ & $(\%)$ & $\mathrm{n}(\%)$ & $\mathrm{n}(\%)$ \\
\hline Hematological & & & & & & \\
Anemia & 25 & $(53.2)$ & 4 & $(8.5)$ & 29 & $(61.7)$ \\
Neutropenia & 24 & $(51.1)$ & 4 & $(8.5)$ & 28 & $(59.6)$ \\
Thrombocytopenia & 22 & $(46.8)$ & 3 & $(6.4)$ & 25 & $(53.2)$ \\
Non-hematological & & & & & & \\
Fatigue & 13 & $(27.6)$ & 2 & $(4.3)$ & 15 & $(31.9)$ \\
Neuropathy & 7 & $(14.9)$ & 1 & $(2.1)$ & 8 & $(17)$ \\
Mucosit & 6 & $(12.8)$ & 0 & 0 & 6 & $(12.8)$ \\
Vomiting & 6 & $(12.8)$ & 4 & $(8.5)$ & 10 & $(21.3)$ \\
Hand-foot syndrome & 12 & $(25.5)$ & 1 & $(2.1)$ & 13 & $(27.6)$ \\
Nause & 11 & $(23.4)$ & 5 & $(10.6)$ & 16 & $(34.0)$ \\
Diarrhea & 9 & $(19.1)$ & 2 & $(4.3)$ & 11 & $(23.4)$ \\
\hline a National Cancer Institute & & & & & &
\end{tabular}

(range, 3-77000) and there were no correlation between baseline CA 19-9 levels and overall survival of the patents.

\section{Toxicity}

The most common reason for treatment withdrawal was disease progression $(n=26 ; 55 \%)$. Dose reductions were required in 16 of $47(34 \%)$ patients (both for first cycle of chemotherapy due to ECOG 2 with comorbidities and after grades 3-4 toxicity). The most common grade 3-4 toxicities were nausea, vomiting and hematologic side effects. Table 3 lists the common treatment-related toxicities. When we compared the survival outcome of the patients with respect to dose reduction (yes $v s$ no), there were no significant differences between these groups.

\section{Discussion}

Pancreatic cancer is among the most challenging of solid malignancies to treat. Most patients die from the disease because of its propensity for late presentation with advance stage, aggressive tumor biology and resistance to chemotherapy. When we evaluated XELOX in second-line treatment, we found it is effective for patients who were progressed after first-line gemcitabine-based therapy. Treatment was well tolerated and resulted in a median overall survival of 23 weeks with a 6-month survival rate of $40 \%$ and median progression-free survival was 12 weeks.

In daily practice, patients who are treated with chemotherapy have good performance status and only some of them are treated with salvage therapy after disease progression. This may be largely due to the fact that many patients have a declining performance status and are no longer eligible to receive further chemotherapy. In addition, after failure of first-line therapy, there are limited data to support a standard second-line chemotherapy regimen for advanced pancreatic cancer and the efficacy and benefit of salvage treatments in terms of survival or quality of life is not clear.

Median survival is approximately 2 months for the patients treated with best supportive care after first-line chemotherapy (Pelzer et al., 2011). Several clinical trials have evaluated the safety and efficacy of second-line chemotherapy in this patient population (Klapdor et al., 2000; Mitry et al., 2006; Choi et al., 2014). Guidelines for pancreatic carcinoma currently recommend second-line chemotherapy in selected patients based on the previous publications in which the overall survival is in the range of 3 to 8 months (Demols et al., 2006; Reni et al., 2006; Ignatiadis et al., 2006; Mitry et al., 2006; Boeck et al., 2007; Kulke et al., 2007). Kim et al. (2012) analyzed 90 advanced pancreatic adenocarcinoma patients who had received second-line chemotherapy after failure of firstline therapy and they tried to develop a prognostic model to identify patients who would benefit from second-line treatment. In that study, all enrolled patients have ECOG performance status $0-2$. The researchers concluded that good performance status (PS: 0-1) and response to firstline treatment were predictive factors to select cases where second-line therapy would be beneficial. Herrmann et al. (2007) had analyzed 46 metastatic patients who had progressed following first-line therapy. Time to progression (TTP) $<6$ months was shown to be strong and highly significant independent prognostic factor for residual survival just like our study.

The only established therapy was 5-flouorouracil, leucovorin and oxaliplatin (FOLFOX) combination, according to the results from the phase III CONKO-003 trial. The results of this study showed significant improvements in both median PFS (13 vs 9 weeks; $\mathrm{p}=0.012)$ and $\mathrm{OS}$ (20 vs 13 weeks; $\mathrm{p}=0.014)$ when oxaliplatin was added to 5-fluorouracil/leucovorin and making this regimen the standard approach for second-line therapy in metastatic pancreatic cancer patients (Pelzer et al., 2011). In our study, we evaluated the combination of oxaliplatin with capecitabine instead of continuous 5-FU infusion. Treatment related toxicity and the survival results were similar with CONKO 003 trial in terms of both median PFS (12 vs 13 weeks) and OS (23 vs 20 weeks).

Capecitabine, an oral tumor-selective fluoropyrimidine, has been reported to have comparable efficacy to infusional 5-FU (Reigner et al., 2001). In a phase II trial done by Xiong et al, 41 patients who have received oxaliplatin plus capecitabine treatment were evaluated. There was one partial response and 22\% clinical benefit rate. Median PFS was 10 weeks, and 6- and 12-month survival rates were $44 \%$ and $21 \%$, respectively (Xiong et al., 2008). They have already shown the benefit of oxaliplatin in combination with capecitabine and our results are also correlated with this study. In a retrospective analysis, Tsavaris et al. (2005) had showed the potential effectiveness of second-line therapy with FOLFOX following confirmed progression with a gemcitabine-containing schedule. Among 30 patients, they reported PR in 7 (23.3\%), and stabile disease (SD) in $9(30 \%)$ patients. In present study, 8 (17.0\%) patients had PR and 10 patients (21.3\%) had SD.

Despite recent advances in cancer management, the subject of selecting a second-line regimen after gemcitabine-failure remains controversial for pancreatic cancer patients. We evaluated the combination of oxaliplatin with capecitabine and found similar results to other second-line regimens. We concluded that XELOX is an effective second-line combination for metastatic 
pancreatic cancer patients with manageable toxicity.

\section{References}

Boeck S, Weigang-Kohler K, Fuchs M, et al (2007). Second-line chemotherapy with pemetrexed after gemcitabine failure in patients with advanced pancreatic cancer: a multicenter phase II trial. Ann Oncol, 18, 745-51.

Burris HA, III, Moore MJ,Andersen J et al (1997). Improvements in survival and clinical benefit with gemcitabine as first- line therapy for patients with advanced pancreas cancer: a randomized trial. J Clin Oncol, 15, 2403-13.

Canyilmaz E, Serdar L, Uslu GH, et al (2013). Evaluation of prognostic factors and survival results in pancreatic carcinomas in Turkey. Asian Pac J Cancer Prev, 14, 6573-8.

Chen WQ, Liang D, Zhang SW, et al (2013). Pancreatic cancer incidence and mortality patterns in china, 2009. Asian Pac J Cancer Prev, 14, 7321-4.

Cheung R (2013). Racial and social economic factors impact on the cause specific survival of pancreatic cancer: a SEER survey. Asian Pac J Cancer Prev, 14, 159-63.

Choi JG, Seo JH, Oh SC, et al (2012). A Phase II Trial of Gemcitabine plus Capecitabine for Patients with Advanced Pancreatic Cancer. Cancer Res Treat, 44, 127-32.

Choi M, Saif MW, Kim R (2014). Is there a role for second line therapy in advanced pancreatic cancer? JOP, 15, 106-9.

Conroy T, Desseigne F, Ychou M, et al (2011). FOLFIRINOX versus gemcitabine for metastatic pancreatic cancer. The New England Journal of Medicine, 364, 1817-25

Demols A, Peeters M, Polus M, et al (2006). Gemcitabine and oxaliplatin (GEMOX) in gemcitabine refractory advanced pancreatic adenocarcinoma: a phase II study. Br J Cancer, 94, 481-5.

Díaz-Rubio E, Evans TR, Tabemero Jet al (2002). Capecitabine (Xeloda) in combination with oxaliplatin: a phase I, doseescalation study in patients with advanced or metastatic solid tumors. Ann Oncol, 13, 558-65.

Eisenhauer EA, Therasse P, Bogaerts J, et al (2009). New response evaluation criteria in solid tumours: revised RECIST guideline (version 1.1). Eur J Cancer, 45, 228-47.

Faivre S, Raymond E, Woynarowski JM, et al (1999). Supraadditive effect of 2',2' difluorodeoxycytidine (gemcitabine) in combination with oxaliplatin in human cancer cell lines. Cancer Chemother Pharmacol, 44, 117-23.

Fernandez E, La Vecchia C, Porta M, et al (1994). Trends in pancreatic cancer mortality in Europe, 1955-1989. Int $J$ Cancer, 57, 786-92

Fesinmeyer MD, Austin MA, Li CI, et al (2005). Differences in survival by histologic type of pancreatic cancer. Cancer Epidemiol Biomarkers Prev, 14, 1766-73.

Glimelius B, Hoffman K, Sjoden PO, et al (1996). Chemotherapy improves survival and quality of life in advanced pancreatic and biliary cancer. Ann Oncol, 7, 593-600.

Herrmann C, Abel U, Stremmel W, et al (2007). Short time to progression under first-line chemotherapy is a negative prognostic factor for time to progression and residual survival under second-line chemotherapy in advanced pancreatic cancer. Oncology, 73, 335-9.

Ignatiadis M, Polyzos A, Stathopoulos GP, et al (2006). A multicenter phase II study of docetaxel in combination with gefitinib in gemcitabine-pretreated patients with advanced/ metastatic pancreatic cancer. Oncology, 71, 159-63.

Inal A, Ciltas A, Yildiz R, et al (2012). Long term survivors with metastatic pancreatic cancer treated with gemcitabine alone or plus cisplatin: a retrospective analysis of an Anatolian Society of Medical Oncology multicenter study. Asian Pac J Cancer Prev, 13, 1841-4.
Kim ST, Choi YJ, Park KH, et al (2012). A prognostic model to identify patients with advanced pancreas adenocarcinoma who could benefit from second-line chemotherapy. Clin Oncol (R Coll Radiol), 2, 105-11

Klapdor R, Fenner C (2000). Irinotecan (Campto R): efficacy as third/fourth line therapy in advanced pancreatic cancer. Anticancer Res, 20, 5209-12.

Kulke MH, Blaszkowsky LS, Ryan DP, et al (2007). Capecitabine plus erlotinib in gemcitabine-refractory advanced pancreatic cancer. J Clin Oncol, 25, 4787-92.

Luke C, Price T, Karapetis C, et al (2009). Pancreatic cancer epidemiology and survival in an Australian population. Asian Pac J Cancer Prev, 10, 369-74.

Mitry E, Ducreux M, Ould-Kaci M, et al (2006). Oxaliplatin combined with 5- FU in second line treatment of advanced pancreatic adenocarcinoma. Results of a phase II trial. Gastroenterol Clin Biol, 30, 357-63.

Neoptolemos JP, Cunningham D, Friess H, et al (2003). Adjuvant therapy in pancreatic cancer: historical and current perspectives. Ann Oncol, 14, 675-92

Oken MM, Creech RH, Tormey DC, et al (1982). Toxicity and response criteria of the Eastern Cooperative Oncology Group. Am J Clin Oncol, 6, 649-55.

Pelzer U, Schwaner I, Stieler J, et al (2011). Best supportive care (BSC) versus oxaliplatin, folinic acid and 5-fluorouracil (OFF) plus BSC in patients for second-line advanced pancreatic cancer: a phase III-study from the German CONKO-study group. Eur J Cancer, 47, 1676-81

Qureshi A, Hassan U, Azam M. (2011). Morphology, TNM staging and survival with pancreatico-duodenectomy specimens received at Shaukat Khanum Memorial Cancer Hospital and Research Centre, Pakistan. Asian Pac J Cancer Prev, 12, 953-6.

Reigner B, Blesch K, Weidekamm E (2001) Clinical pharmacokinetics of capecitabine. Clin Pharmacokinet, 40, 85-104.

Reni M, Pasetto L, Aprile G, et al (2006). Raltitrexed-eloxatin salvage chemotherapy in gemcitabine-resistant metastatic pancreatic cancer. Br J Cancer, 94, 785-91.

Siegel R, Naishadham D, Jemal A (2013). Cancer statistics, 2013. CA Cancer J Clin, 63, 11-30

Tokh M, Bathini V, Saif MW (2012). First-line treatment of metastatic pancreatic cancer. JOP, 13, 159-62

Trotti A, Colevas AD, Setser A, et al (2003). CTCAE v3.0: development of a comprehensive grading system for the adverse effects of cancer treatment. Semin Radiat Oncol, 13,176-81.

Tsavaris N, Kosmas C, Skopelitis H, et al (2005). Second-line treatment with oxaliplatin, leucovorin and 5-fluorouracil in gemcitabine-pretreated advanced pancreatic cancer: A phase II study. Invest New Drugs, 23, 369-75.

Von Hoff DD, Goodwin AL, Garcia L (1998). Advances in the treatment of patients with pancreatic cancer: Improvement in symptoms and survivial time. Br J Cancer, 78, 9-13

Xiong HQ, Varadhachary GR, Blais JC, et al (2008). Phase 2 trial of oxaliplatin plus capecitabine (XELOX) as secondline therapy for patients with advanced pancreatic cancer. Cancer, 113, 2046-52

Zahir S, Arjmand A, Kargar S, et al (2013). Incidence and trends of malignant and benign pancreatic lesions in Yazd, Iran between 2001 and 2011. Asian Pac J Cancer Prev, 14, 2631-5. 